Mémoires du livre

Studies in Book Culture

\title{
Arriver dans la colonie : distance et littérature. Variations autour d'un motif sous la Troisième République, du journal au livre
}

\section{Laure Demougin}

Volume 10, numéro 1, automne 2018

Voyager dans la modernité : des moyens techniques aux supports imprimés de l'aventure

Adventure: From Travel Technology to Print Media

URI : https://id.erudit.org/iderudit/1055404ar

DOI : https://doi.org/10.7202/1055404ar

Aller au sommaire du numéro

Éditeur(s)

Groupe de recherches et d'études sur le livre au Québec

ISSN

1920-602X (numérique)

Découvrir la revue

Citer cet article

Demougin, L. (2018). Arriver dans la colonie : distance et littérature. Variations autour d'un motif sous la Troisième République, du journal au livre. Mémoires du livre / Studies in Book Culture, 10(1). https://doi.org/10.7202/1055404ar

\section{Résumé de l'article}

Quelles sont les modalités qui permettent au lectorat de l'époque coloniale d'entrer, littérairement parlant, dans l'empire colonial français? Sous la Troisième République, la question est importante pour des raisons politiques et idéologiques : on verra ici, à travers trois cas particuliers, comment les entrées dans les textes engagent différemment l'expérience d'une lecture exotique. Comparer ces premières lignes issues du livre ou des périodiques permet de mettre en évidence les variations de l'aventure coloniale et de sa modernité par le biais de la vitesse narrative : un récit de voyage, un roman d'aventure de Jules Verne et un reportage d'Albert Londres trouveront ainsi des points communs qui éclairent l'entreprise coloniale. Selon que les récits ont été publiés dans des journaux ou dans des livres, la vitesse de lecture n'est pas la même, le support jouant un rôle considérable dans la perception de l'aventure elle-même.

Tous droits réservés ( G Groupe de recherches et d'études sur le livre au Québec, 2019

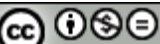




\title{
MÉMOIRES DU LIVRE \\ STUDIES IN BOOK CULTURE
}

\section{ARRIVER DANS LA COLONIE : DISTANCE ET LITTÉRATURE. Variations autour d'un motif sous la Troisième République, du journal au livre}

\author{
Laure DEMOUGIN \\ Université Paul-Valéry Montpellier III / Université Laval
}

Quelles sont les modalités qui permettent au lectorat de l'époque coloniale d'entrer, littérairement parlant, dans l'empire colonial français? Sous la Troisième République, la question est importante pour des raisons politiques et idéologiques : on verra ici, à travers trois cas particuliers, comment les entrées dans les textes engagent différemment l'expérience d'une lecture exotique. Comparer ces premières lignes issues du livre ou des périodiques permet de mettre en évidence les variations de l'aventure coloniale et de sa modernité par le biais de la vitesse narrative : un récit de voyage, un roman d'aventure de Jules Verne et un reportage d'Albert Londres trouveront ainsi des points communs qui éclairent l'entreprise coloniale. Selon que les récits ont été publiés dans des journaux ou dans des livres, la vitesse de lecture n'est pas la même, le support jouant un rôle considérable dans la perception de l'aventure elle-même.

How can readers of the colonial era enter the French colonial empire through literature? Under the Third Republic, this question matters for political and ideological reasons. This article aims to examine, through three particular case studies, how the first lines of a text engage the experience of an exotic reading differently. Comparing the first lines of a book or of magazines makes it possible to highlight variations in the colonial adventure, and in its modernity, through the speed of the narrative: a travel narrative, a Jules Verne adventure novel, a report by Albert Londres, all possess similarities which shed light on the "colonial adventure." Depending on whether the travel narratives were published in newspapers or in books, the speed of reading is not the same, the medium playing a significant role in the perception of the adventure itself. 
Comment faire entrer le lectorat français de l'époque coloniale, littérairement parlant, dans l'empire colonial, selon qu'il emprunte la porte du récit périodique ou du recueil? Comment peut-on lire les premières lignes des ouvrages qui présentent une arrivée dans les colonies, et selon quel modèle s'opère la coïncidence de l'entrée dans la lecture et de l'arrivée sur le territoire colonial? Car arriver dans la colonie n'est pas seulement une expérience vécue : c'est également une expérience que l'on raconte, dont l'on prévoit le rythme de parution (épisodiquement dans un journal, ou sous la forme d'un livre?); c'est enfin, sous la Troisième République qui a fait de la colonisation un pilier de son action politique, un moment fort des récits coloniaux ${ }^{1}$. Entre 1870 et 1930, l'empire colonial français connait en effet une expansion remarquable; plus généralement, le lien entre la colonisation et l'idéologie du progrès se manifeste tout au long du XIX ${ }^{e}$ siècle, et particulièrement avec le «moment colonial ${ }^{2}$ » de la Troisième République qui théorise une colonisation aux motivations dépassant le cadre strictement économique. Dans cette perspective, la colonisation met en place une perception linéaire du temps, orientée vers le progrès ${ }^{3}$. Le rythme colonial, au sens large, est marqué par cette pensée de l'avenir qui se réalise également dans le domaine culturel. S'inventent ou s'affirment alors de nouvelles formes littéraires, publiées sur de nouveaux supports, qui font entrer en jeu de nouvelles images d'auteurs : le reporter devient ainsi l'une des figures marquantes d'un champ littéraire plus largement configuré par une forte présence journalistique ${ }^{4}$.

Le support de publication joue un rôle important dans l'écriture de l'arrivée dans une colonie : comparer les premières lignes de ces publications entre la presse et le livre, entre la fin du XIX siècle et le début du XXe siècle permet donc d'envisager les modifications du rapport à l'aventure et à sa modernité par le biais des moyens de transport. Plus largement, vitesse d'écriture et vitesse de lecture se recoupent : nous nous proposons ici de les lier à la question du support de publication et à la perception de la modernité du voyage. En d'autres termes, l'accélération pragmatique des déplacements et des échanges a-t-elle eu comme répercussion une accélération de l'entrée dans la lecture? Une telle question constitue un axe d'étude pour examiner sous un même angle des textes différents, réunis par leur traitement d'un territoire colonial. L'aspect empirique de la lecture reste inaccessible; mais les rythmes de l'écriture occasionnent des effets de seuil qui sont, quant à eux, perceptibles. Les répercussions de la publication périodique sur les rythmes 
de l'écriture, partant sur les rythmes de lecture, forment une perspective pour la relecture des textes journalistiques ${ }^{5}$. Ainsi, dans un effort de distinction générique, « [tout] un rapport au temps (temps stratifié, temps archéologique, temps de la publication) sépare récit de voyage et reportage ${ }^{6} »$ : c'est à partir de ces premières remarques que peut s'esquisser une étude de la manière dont on écrit (et dont on lit) les voyages coloniaux selon qu'ils paraissent dans le journal ou en recueil. La narration du voyage connait à cette période des transformations stylistiques et éditoriales : les récits se donnent à lire dans la presse sous la forme de ce qui devient un reportage, mettant en avant une subjectivité qui se veut elle aussi moderne, répondant à une temporalité coloniale obnubilée par le temps présent et le progrès, par son besoin d'actualité 7 . Ainsi, « au passé de la littérature, comme aux mirages de la fiction, le reporter oppose le présent d'un référent dont il décuple la présence par l'exercice d'un regard ${ }^{8} »$.

Il ne s'agit pas ici d'étudier statistiquement les représentations de l'arrivée sur les rivages coloniaux, mais plutôt de prendre quelques cas signifiants pour retracer, mutatis mutandis, l'écriture de l'arrivée coloniale, depuis un récit de voyage publié en 1871 jusqu'à un reportage de 1928. L'auteur d'un voyage en Guyane, au XIXe siècle, ne traite pas de la même manière le début de l'aventure selon qu'il publie dans La Fenille de la Guyane française ou en recueil chez un libraire nantais. Si l'on élargit la perspective, et bien que le texte n'ait pas varié entre sa publication périodique et sa publication en recueil, les héros verniens de Clovis Dardentor, en 1896, n’arrivent sur le territoire algérien qu'après quelques pages, dans un incipit déceptif qui joue avec l'attendu voulant que le début de la lecture corresponde au début du voyage. Plus tard dans la chronologie coloniale, enfin, l'arrivée au Sénégal n'est pas tout à fait la même dans le reportage d'Albert Londres paru en 1928 dans Le Petit Parisien, d'abord intitulé «Quatre mois parmi nos noirs d'Afrique », puis Terre d'ébène quand il paraît en recueil chez Albin Michel : si le texte a peu varié, les conditions de lecture, la préface, l'environnement du texte en font cependant deux objets littéraires différents. Les extraits présentés ici ont en commun de décrire des arrivées, d'inaugurer des épisodes qui mèneront à l'exploration d'un territoire appartenant aux colonies françaises. S'y croisent des faits stylistiques, idéologiques, littéraires au sens large: mettant de côté les questions génériques, le présent article a pour but d'interroger les liens entre le rythme de la lecture et l'histoire matérielle des publications, d'envisager, autrement 
dit, les variations du pacte de lecture initial selon les temporalités liées aux supports de publication. Ouvrir l'œuvre par un déplacement synonyme de rapidité participe à l'inscription dans la modernité d'une forme de littérature réaliste, affiche sa référentialité: les variations que nous étudierons ici tiendront donc aux différences entre livre et journal, entre recueil et périodique.

\section{Un voyage en Guyane : du journal colonial au recueil grâce à la goélette}

Un texte paraît du 27 mai au 17 juin 1871 dans La Fexille de la Guyane française (périodique officiel publié à Cayenne) qui s'intitule «Voyage chez les Indiens de la Guyane ». Le lecteur ne se trouve pas ici dans le cadre d'un reportage : c'est un récit de voyage qu'annonce la rédaction, lequel est déjà ancien au moment de sa publication dans le périodique. Dans la version journalistique de 1871, l'aventure est datée de 1863; dans la version de librairie de 1886, elle est datée de 1865 : dans les deux cas, il y a bien un écart temporel qui justifie l'appartenance au récit de voyage. Quand le récit parait en feuilleton, c'est sous la signature d'Arthur Delteil, que la notice de la Bibliothèque Nationale signale comme un pharmacien de $1^{\mathrm{e}}$ classe de la Marine et chevalier de la Légion d'honneur (1835-1905), et que la rédaction du périodique prend la peine de présenter comme suit dans un paragraphe introductif à l'article :

Nous devons à l'obligeance de M. Couy, Maire de Cayenne, et Président de la Chambre d'agriculture, de commerce et d'industrie, la communication du très-intéressant document que nous publions ci-après. Nous saisissons cette occasion de remercier M. Delteil du bon souvenir qu'il a conservé de la Guyane, nonobstant les occupations nombreuses que lui imposent, à la Réunion, sa position de chef du service pharmaceutique ${ }^{9}$.

L'auteur est en quelque sorte autorisé à parler des colonies, puisqu'il appartient lui-même aux cadres coloniaux; le texte est défini en termes catégoriels assez précis : c'est un "document» présentant un "souvenir », donc affichant une distance à la fois temporelle et émotionnelle. Le récit peut 
alors commencer à proprement parler, et le chef du service pharmaceutique de relater :

À la suite de l'assassinat commis par un métis brésilien sur un de mes oncles, qui vivait depuis plusieurs années au milieu des tribus indiennes, presqu'au centre de la Guyane, mon beau-père se trouva dans la nécessité de se rendre sur l'établissement jadis occupé par son malheureux frère, afin de recueillir les quelques restes de son modeste héritage ${ }^{10}$.

C'est par le fait divers remémoré que le voyage débute; les motivations du voyageur sont développées dans le paragraphe suivant, qui est de l'ordre de la pause narrative: le devoir familial, mais également la curiosité pour les paysages et les «tribus indiennes qui ne se rencontrent plus que dans ces régions éloignées et inaccessibles ${ }^{11}$ ", sont ainsi mentionnés. À cette introduction explicative, sorte de faux départ dans le voyage, succède enfin la mention du trajet:apparaissent alors Cayenne directement (et non La Réunion, d'où vient le voyageur), et la silhouette de la goélette, ce «navire léger à deux mâts et à voiles auriques ${ }^{12}$ » qui peuple les récits coloniaux et que les lecteurs contemporains connaissent bien.

Une fois tous nos préparatifs terminés, nous partîmes par un beau matin du mois de septembre 1863, sur une goélette, fine voilière, qui faisait les voyages de Cayenne à Guisambourg, petit poste situé à l'embouchure de l'Approuague ${ }^{13}$.

Le rythme du récit ralentit ensuite: le voyageur présente au lecteur ses compagnons et adapte le récit de la traversée à la cadence des conversations, avec quelques variations qui tiennent à la nature du «souvenir» raconté (il mentionne ainsi être resté ami «depuis » avec l'un de ses compagnons de voyage). L'auteur, ici, joue donc à la fois sur le caractère relativement lointain des événements racontés, et sur la reconstitution du rythme - lent, malgré quelques ellipses - du voyage sur le fleuve par le biais des discours rapportés.

Pendant que notre navire, poussé par une jolie brise, glissait au milieu des îles verdoyantes parsemées çà et là le long des palétuviers de la côte, je provoquai, pour passer le temps, ce silencieux et flegmatique personnage, à me faire des confidences; au bout de quelques heures, je connaissais toute l'histoire aventureuse de sa vie, et une mutuelle sympathie préludait déjà à 
la solide et inaltérable amitié qui nous a liés depuis. - Comme tous les hommes de cœur de son pays, il avait voulu prendre part à la dernière révolution dans laquelle la Pologne tentait de secouer une fois encore le joug de ses oppresseurs ${ }^{14}$.

Ces variations sont la clef d'une écriture qui, parce qu'elle est occasionnelle et non professionnelle, adopte les canons d'un genre qui est celui du voyage colonial. Donner à lire le mouvement de la goélette fait partie des canons d'écriture; rythmer ce trajet par les discours rapportés en constitue un autre aspect. Ainsi d'un second passage :

Mais pendant que nous bavardons, la nuit est arrivée, notre goëlette [sic] entre dans l'embouchure de l'Approuague; les bords du fleuve se dessinent vaguement au milieu de l'obscurité : pour plus de sûreté, notre patron se décide à jeter l'ancre, afin d'éviter les bancs de vase sur lesquels nous aurions pu nous échouer en continuant notre navigation ${ }^{15}$.

Delteil fera de même pour les quelques événements ponctuant le trajet fluvial, ralentissant alors le tempo du récit par des scènes plus longues. Quand les voyageurs quittent le bateau pour la pirogue, la description, en revanche, augmente en vitesse. La goélette symbolise l'entrée dans l'aventure coloniale : on y discute encore selon le rythme de la société coloniale avant de passer aux aventures des territoires éloignés des rivages, inconnus du grand public, le passage à la pirogue signalant une accélération de l'aventure et la fin du confort colonial. Le lectorat de La Feuille de la Guyane française, qu'il soit métropolitain (par échange, correspondance, abonnement) ou guyanais, est urbain : glisser le long du fleuve sur la goélette permet au lecteur de prendre conscience de son entrée progressive sur le territoire inconnu de la forêt.

Ce récit de voyage est assez représentatif des divers rapports de voyages, explorations, promenades et autres déplacements protéiformes que l'on peut trouver dans la presse coloniale. Mais il a ceci d'original qu'il est republié plus tard sous forme de recueil et avec de nombreuses corrections. En 1886, le voyage d'Arthur Delteil paraît en effet en livre chez un éditeur nantais, L. Mellinet; le titre n'a pas changé, mais le texte, quelque peu. Passer de la publication dans un journal colonial officiel à une publication en recueil nécessite, a priori, de repenser les rythmes de lecture et le lectorat en tant que tel. Ce n'est pas, en outre, une tâche facile : il a ainsi fallu 15 ans à Delteil pour 
faire éditer son voyage guyanais. Entretemps, le pharmacien de marine a rédigé plusieurs ouvrages documentaires et coloniaux : une Étude sur la vanille, un ouvrage sobrement intitulé La Canne à sucre, une Étude sur le climat de la Cocbinchine, une Note sur le climat de la Réunion ${ }^{16}$. Se sont donc développées conjointement son appartenance au monde colonial et sa capacité à produire des textes scientifiques et sérieux qui s'éloignent le plus possible de l'aventure. D'où, peut-être, un changement de ton dans les premières lignes de ce récit qu'il reprend :

Le 2 septembre 1865, je partais de Cayenne sur une goélette qui devait me conduire à Guisanbourg, poste situé à l'embouchure de l'Approuague, un des plus grands fleuves de la Guyane. J'avais pour compagnons de voyage un vieil habitant de Cayenne, habitué aux excursions lointaines dans l'intérieur des grands bois, et un naturaliste polonais, nommé Yelski, qui arrivait tout récemment de France et se proposait de parcourir les forêts de la Guyane pour y faire d'importantes collections. L'objectif de notre voyage était de visiter l'Approuague, de remonter l'Arataye, un de ses affluents, et de pousser jusqu'aux régions habitées par les Indiens-Galibis. C'était un projet caressé depuis longtemps; j’allais donc pouvoir admirer les splendides merveilles de la végétation des grands bois et étudier d'après nature les mœurs des tribus indiennes qui ne se rencontrent plus que dans l'intérieur de notre possession; je me faisais une véritable fête de voir de près ces premiers possesseurs du sol, dont l'ardeur belliqueuse avait tenu si souvent en échec les Européens qui s'étaient établis, il y a deux siècles, à Cayenne.

Notre petit navire, poussé par une jolie brise, glisse au milieu des îles verdoyantes parsemées çà et là le long de la côte de l'île de Cayenne, et qui ont reçu les noms d'Ilet-la-Mère, d'Ilet-le-Père, des Deux-Mamelles; nous doublons ensuite la montagne du Diamant, située à l'embouchure du Mahury, et qui ressemble de loin à une pyramide quadrangulaire tronquée. Puis notre vue ne s'étend plus que sur des savanes noyées, des côtes basses et marécageuses des pays de Kaw, garnies de palétuviers, et qui ne sont habitées que par d'énormes caïmans, des tortues monstrueuses et des boas gigantesques. Ce sont des continents en formation qui s'élèvent d'année en année, à la suite des apports alluvionnaires laissés par les inondations.

À la tombée de la nuit, notre goélette entre dans l'embouchure de l'Approuague, les bords du fleuve se dessinent vaguement au milieu de l'obscurité : pour plus de sûreté, notre patron se décide 
à jeter l'ancre, afin d'éviter les bancs de vase sur lesquels nous aurions pu nous échouer en continuant notre navigation ${ }^{17}$.

Plus de discours rapportés, plus de coïncidence entre la lecture et le trajet de la goélette - qui, en 15 ans, est bien devenue le " petit » navire déjà marqué par la désuétude, puisque les voiliers sont remplacés par les bateaux à vapeur (les steamers de la modernité) : le récit est donc réécrit en coupant ce qui faisait participer le lecteur au rythme lent de la remontée du fleuve. La survenue du présent de narration pour regagner le rythme de l'actualité est éclipsée par un présent qui recouvre tout le texte, et le récit gagne en effet en efficacité ce qu'il perd en travail sur la temporalité : il se fait moins digressif et passe outre la justification du voyage (le meurtre initial et le devoir familial) pour forger une autre image de l'auteur.

Ce que la publication en recueil a gommé, c'est donc l'actualité qu'introduit le motif donné aux lecteurs du journal, et qui oriente la lecture dans la direction d'une forme d'aventure induite par la mention du meurtre initial. Plus personnel, le récit de la presse coloniale offre également plus de prise à la représentation du danger, car il s'agit de gagner une forme de légitimité par la justification du déplacement. Delteil publiant dans La Feuille de la Guyane française n'est pas un simple touriste, mais bien un colonial passé d'un territoire à un autre pour des raisons familiales. Dans la parution en livre, oublier cette justification et faire débuter le récit par la traversée en goélette revient à mettre en relief le voyage avant tout, et non sa justification territorialisée : c'est montrer l'auteur en savant curieux, et non en amateur d'aventures dont le voyage était nécessaire. Si l'on raisonne en termes de supports, le pacte de lecture journalistique s'établit sur une forme de ressemblance, engageant la possibilité d'une cooptation par les coloniaux qui se reconnaissent dans les colonnes familières de leur journal. Le pacte de lecture livresque s'appuie davantage sur la topique de la traversée inaugurale, promesse de découverte à venir, scène accessible au grand public et document neutre pour les lecteurs.

\section{Un voyage en Algérie : Jules Verne, entre le train et le paquebot}

Les liens entre le journal et le recueil sont multiples, et ils ont été étudiés plus largement ${ }^{18}$. Or il existe d'autres liens entre les deux supports qui peuvent 
faire émerger des temporalités diverses. Ainsi de Jules Verne : l'auteur emblématique des «romans géographiques ${ }^{19}$ » garde toujours un rapport particulier aux publications journalistiques, et à l'objet même que représente le journal, puisque ses romans se terminent fréquemment sur l'évocation d'un journal ${ }^{20}$. Verne se situe bien aux premières loges de ce que les évolutions technologiques ont pu apporter à l'écriture : les moyens de se déplacer et d'informer se trouvent au centre de ses publications, et ses romans sont rythmés par sa relation à l'actualité et à la mondialisation ${ }^{21}$. Il a cependant peu écrit sur les colonies françaises; et l'un de ses romans dont l'action se déroule sur le territoire colonial n'est pas le plus connu: Clovis Dardentor, roman éponyme de 1896, a échappé à la postérité22. Quand il parait, il est annoncé dans le supplément du 20 décembre 1896 du Temps en ces termes : «Clovis Dardentor est un récit d'aventures de voyages d'une irrésistible et communicative bonne humeur ${ }^{23} »$. Le nouveau livre d'étrennes du prolifique auteur des Voyages extraordinaires a en effet ceci d'original qu'il est humoristique et algérien. Ces particularités ressortent d'autant plus quand l'on sait que Clovis Dardentor parait chez Hetzel en même temps que Face au drapeau. Alors que Clovis Dardentor joue la carte de l'humour, Face an drapean présente un personnage de savant français, Thomas Roch, d'abord interné après avoir perdu la raison, puis enlevé par de modernes pirates pour s'assurer le secret de fabrication de sa bombe. L'ingénieur Simon Hart, qui accompagne Roch en se faisant passer pour son infirmier, apparait au centre de l'intrigue, ses notes constituant la plus grande partie du roman. Par sa profession, il représente la France du progrès; par son patriotisme, la France face aux autres puissances mondiales : et la fin du roman, qui voit Thomas Roch retrouver sa raison devant l'apparition du drapeau français, montre assez la différence de tonalité qui sépare les deux romans de Verne. Les épisodes coloniaux de Clovis Dardentor, bien qu'empreints de danger, restent relatés sur un ton plus léger : il y est question de mariage et d'adoption, de petites lâchetés, et d'excursions touristiques en train.

Puisque les deux romans sont parus la même année et ont été présentés au public par les mêmes paragraphes dans les journaux, il est tentant de comparer rapidement leurs premières lignes. Face au drapean commence avec la réception d'une carte mystérieuse par «le directeur de l'établissement de HealthfulHouse $^{24}$ » et met en place une tension narrative forte dès cette première action. Les aventures de Clovis Dardentor, au contraire, s'amorcent de manière 
bien plus déceptive et bien plus coloniale, puisque deux jeunes gens (Marcel Lornans et Jean Taconnat) descendent d'un train en attendant d'embarquer pour Alger. Le personnage éponyme est donc absent de ces premières pages, comme le précise l'intitulé du premier chapitre, «Dans lequel le principal personnage de cette histoire n'est pas présenté au lecteur ${ }^{25} »$. Les jeunes voyageurs verniens qui se rendent en Algérie sont saisis, non au moment de l'embarquement vers les trois départements français, mais dans un temps mort du voyage, celui d'une correspondance. Les héros ne montent pas dans le paquebot, ce qui serait un geste dynamique : ils descendent en gare de Sète - de Cette, selon l'orthographe de l'époque - et attendent la suite de leur aventure en «[récitant] du Joanne ${ }^{26}$ » comme tout touriste qui s'informe. Le mouvement de la découverte est détourné, la scène placée sous la perspective du burlesque : les deux touristes offrent une version évaporée de l'aventurier. Cela correspond à la fois à la vocation touristique de l'Algérie telle que l'organise le gouvernement français, et à la difficulté de faire perdurer l'aventure en cette fin de siècle. Littérairement, l'humour vernien se révèle ici par sa gestion de la temporalité, par cette scène initiale qui oppose l'ennui d'une correspondance à la rapidité des transports modernes. Dans une perspective inaugurée par Alphonse Daudet avec la publication des Aventures prodigieuses de Tartarin de Tarascon en 1872, et que les lecteurs de l'époque repèrent, l'incipit vernien parie donc sur l'usure du voyage, malgré les signes de progrès visibles que sont les moyens de transport ${ }^{27}$. Le lecteur est confronté à ce début in medias res :

Lorsque tous les deux descendirent en gare de Cette, - train de Paris à la Méditerranée - Marcel Lornans, s'adressant à Jean Taconnat, lui dit:

«Qu'allons-nous faire, s'il te plait, en attendant le départ du paquebot? ...

Rien, répondit Jean Taconnat.

Cependant, à s'en rapporter au Guide du Voyageur, Cette est une ville curieuse, bien qu'elle ne soit pas de haute antiquité, puisqu'elle est postérieure à la création de son port, ce terminus du canal du Languedoc, dû à Louis XIV...

Et c'est peut-être ce que Louis XIV a fait de plus utile pendant toute la durée de son règne! répliqua Jean Taconnat. Sans doute, le Grand Roi prévoyait que nous viendrions nous y embarquer aujourd'hui, 27 avril $1885 \ldots$ 
Sois donc sérieux, Jean, et n'oublie pas que le Midi peut nous entendre! Ce qui me parait sage, c'est de visiter Cette, puisque nous sommes à Cette, ses bassins, ses canaux, sa gare maritime, ses douze kilomètres de quais, sa promenade arrosée par les eaux limpides d'un aqueduc ${ }^{28} \ldots$

L'énumération due au guide touristique, forme d'intertextualité déléguée au personnage, imite les énumérations propres aux romans de Verne. Mais alors que ces énumérations servent habituellement un but pédagogique, c'est une forme de pastiche que l'on lit dans ce ralentissement du rythme qui n'est pas instructif mais humoristique. La réponse donnée par Jean au « qu’allons-nous faire? » de son ami est à ce titre révélatrice. "Rien », réplique-t-il avant que Marcel ne récite le guide : ces premiers pas vers l'aventure coloniale semblent bien décevants. La spécificité même de ce roman dans la production de son auteur pourrait ainsi tenir au thème traité : le voyageur moderne, de train en paquebot, n'est qu'un aventurier raté.

Jules Verne inaugure donc son roman "algérien » par une scène de mise en abyme:le lecteur lit ce que lisent d'autres lecteurs; il ne trouve pas d'accélération liée aux moyens de transports modernes, pas de mystère ni d'aventure, mais, au contraire, une forme de redondance à sa curiosité (de lecteur). Clovis Dardentor parait d'abord dans Le Magasin d'éducation et de récréation : il garde les traces de cette publication périodique, mais reste avant tout une œuvre de recueil, bien loin du feuilleton évoqué précédemment. En pleine Troisième République, les aventures de Marcel Lornans et Jean Taconnat montrent comment le rythme de la lecture peut prendre appui sur le rythme des voyages pour affirmer une identité littéraire: c'est par la longueur de cette scène d'étape que le lecteur apprend à connaitre le caractère plaisant d'un roman qui ne prend pas la technologie du déplacement au sérieux - rythme bien différent de celui de Face au drapeau, dans lequel un miracle technologique permet à la goélette du comte d'Artigas d'avancer à toute vitesse et sans la force du vent, puisqu'elle est secrètement tractée par un sous-marin. 


\section{Un voyage à Dakar : Albert Londres « touche terre "}

Arriver dans une colonie suppose donc un rythme et une image d'auteur, et ces premiers paramètres suffisent à donner une impulsion au récit du voyage. Qu'en est-il alors des variations possibles entre le journal et le livre pour décrire une même arrivée? Les années 1930 offrent une réponse intéressante à cette question : elles représentent «l'âge d'or du reportage », puisque les grands reporters sont connus, présentés au public en amont des publications périodiques, et que leurs textes sont publiés dans des collections faisant la part belle aux «reportages» ou aux «documents ${ }^{29}$ ». Parallèlement à ces évolutions littéraires, au fur et à mesure du développement de la Troisième République, le goût pour les colonies s'est accru, les années 1930 étant considérées comme l'apogée de l'empire colonial français et de son influence. Or, comme tout apogée, elles marquent également une forme d'usure.

Quand Albert Londres publie « Quatre mois parmi nos noirs d'Afrique » dans Le Petit Parisien, la première livraison du reportage a ainsi été préparée par le dévoilement de la scène qui le justifie, de même que par une carte qui permet au lecteur de se repérer avant de se lancer dans l'expérience de lecture ${ }^{30}$. Le rédacteur Élie-J. Bois, qui signe l'article de présentation, écrit :

Albert Londres, que l'on avait rencontré sur toutes les routes d'Europe, y compris les soviétiques; que les Chinois, les Japonais, les Indochinois, les Turcs, les Syriens, les Marocains, les SudAméricains connaissent, qui révéla dans Le Petit Parisien la véritable Guyane et son bagne, qui avait parcouru presque tout l'univers, mais qui n'avait pas vu l'Afrique Noire [sic], s'embarqua pour la voir. Il l'a vue. Il est revenu. Demain, le Petit Parisien commencera la publication de la série d'articles tour à tour amusants, captivants, pittoresques, émouvants, dramatiques, hallucinants, où il retrace ce qui a empli ses yeux ${ }^{31} \ldots$

La présentation est d'ailleurs étonnante en ce qu'elle est démentie par la première livraison : contrairement à ce qu'annonce le rédacteur, Londres luimême précise, dès les premières lignes de son reportage, avoir « touché Dakar dans le temps ${ }^{32} »$, et il décrit rapidement la nuit qu'il avait passée dans la ville. Ce sont donc bien les éléments d'une mise en scène de l'information que l'on trouve ici, d'une scénographie qui est celle du reporter, fondée notamment 
sur le témoignage ${ }^{33}$. Cette notion de scénographie, tirée des études de Dominique Maingueneau, a été utilisée par Myriam Boucharenc en ce qui concerne le reportage, et l'on peut reprendre les mots de Mélodie SimardHoude, dont les travaux s'attachent à la figure du reporter, pour avoir une idée de sa pertinence dans le cadre présent : « la scénographie est ce lieu pivot, réflexif, où se met en scène un imaginaire du métier, de l'enquête en train de se faire, de l'écriture journalistique ${ }^{34} »$. Plusieurs scénographies permettent de comprendre l'image du reporter : ici, le déplacement est au premier plan, comme le sont également les conditions matérielles qui précèdent l'écriture à proprement parler. Dans le numéro du lendemain, le reportage s'affiche en une avec le sous-titre «En touchant terre à Dakar», seuil de l'aventure que redouble l'exclamation « C'était Dakar! » inaugurant l'accès au vif du sujet. Le gérondif produit une forme de dilatation du moment de l'arrivée pour le lecteur du journal en quête d'actualité : on retrouve ainsi l'idée selon laquelle le reporter «manifesta le recentrage de l'imaginaire du voyage, lequel était consubstantiel au journal depuis le XVIII siècle, autour de ce qui apparaissait désormais comme les deux catégories fondamentales de la presse moderne : la quotidienneté et l'actualité35 ». Le rythme donné par les exclamations initiales correspond particulièrement bien à cette manifestation de l'actualité au sens journalistique, que l'on peut confondre avec l'actualité de la lecture. L'analepse qui suit rapidement le récit du débarquement fait office de ralentissement du rythme narratif. Après la succession des lieux emblématiques vus de la mer (le palais du gouverneur, Gorée), après la brève description des conditions du débarquement, c'est en effet le souvenir qui occupe plusieurs lignes, entretenant la ligne temporelle du récit jusqu'au retour de la réalité : «Six heures! on accroche la passerelle au bateau ${ }^{36}$. » $\mathrm{La}$ digression n'a pas, en fait, été une pause : par ce biais, le reporter a fait patienter son lecteur jusqu'à l'événement important de son récit, le débarquement au sens strict, marqué par le rappel de l'heure - une forme de ponctualité propre aux liaisons maritimes.

Mais que penser des premiers mots du reportage? Lire « C'était Dakar! » dans les colonnes du journal ne procure pas un étonnement au lecteur, mais provoque seulement une accélération de son rythme de lecture par rapport à la présentation initiale du reportage, plus développée, et par rapport au reste de la une dont les articles sont moins rythmés. L'exclamation suscite également une remobilisation des connaissances que le rédacteur lui a fournies 
dans le numéro précédent. Nous nous arrêterons sur cette manière d'inaugurer une étape de son voyage que Londres utilise à plusieurs reprises dans les épisodes du reportage, lesquels forment ainsi autant de petits seuils à franchir quotidiennement pour le lecteur attentif. La livraison du 18 octobre 1928, sous le titre du reportage et une titraille détaillée ("Les inscriptions et les tombes du Soudan, évocatrices de la période héroïque de la conquête »), commence par un seul mot: «Soudan ${ }^{37}$ !» Après un retour à la ligne, le reporter continue : «Dans cette brousse, il vous vient subitement une pensée. Le paysage, les indigènes, cela disparaît un moment à vos yeux. Un écran s'interpose entre vous et cette réalité. Et sur l'écran, on voit : La conquête 38 ! » Le dernier syntagme est mis en valeur par un retour à la ligne : la manière dont le reporter exprime une forme de mirage prend appui sur la lecture quotidienne à laquelle, avec ce rythme haché qui est le sien, il a habitué son lecteur. Dans la publication en recueil, la titraille explicative que nous avons citée disparaît au profit d'une phrase tirée de l'article lui-même : «C'était entre 1880 et $1900{ }^{39}$. » Ce changement de titre opère une entrée accélérée dans le détail du texte : il n'y a plus trace de la structure journalistique nécessaire à l'information, et l'exclamation initiale apparait plus nettement. D'autres passages du reportage, d'autres seuils d'épisodes, sont marqués par ces exclamations : dans le journal, sous la titraille « Dans la forêt en exploitation. Les efforts surhumains des hommes-chevaux - les noirs - et du chef de chantier - un blanc », l'article commence par « La forêt! Le terrifiant royaume des coupeurs de bois $40 ! »$; plus tard, une livraison s'ouvre sur "Ouagadougou ${ }^{41}$ !», ces formules étant conservées dans le livre. Cette forme initiale s'y accentue : l'un des chapitres s'intitule «Tombouctou ${ }^{42}$ ! » alors qu'il était paru dans la presse sous le titre "Tombouctou et sa légende de mystère ${ }^{43}$ ». C'est une preuve de l'intérêt pour les titres courts, saisissants, qui raniment l'attention du lecteur et inaugurent efficacement de nouveaux épisodes du voyage dans le cadre du livre. En se débarrassant du seuil intermédiaire que représente la titraille, le passage au livre a donné au reportage un rythme plus bref, propre à remotiver le tempo de l'aventure pour le lecteur ${ }^{44}$.

Plus généralement, la titraille nombreuse du quotidien laisse place au dépouillement du recueil, rebaptisé à cette occasion Terre d'ébène, dont la lecture est préparée elle aussi par un texte initial qui n'est toutefois plus la présentation d'Élie-J. Bois, mais une préface de Londres lui-même. Ce dernier 
rédige en effet un texte introductif qui répond aux critiques suscitées par la parution du reportage : dans les deux cas, périodique ou recueil, le lecteur entre avec retard dans la colonie, mais l'obstacle qui précède la découverte de Dakar n'est pas de même nature. La présentation enthousiaste du rédacteur a été remplacée par une défense polémique de Londres, qui brocarde les journalistes envoyés par le ministre des Colonies pour vérifier ses propos, tout en maintenant l'actualité de son texte : «À l'heure qu'il est, heure fatale, ces missionnaires débarquent à Dakar ${ }^{45}$. » Le livre ne renonce pas à l'actualité, au contraire : il recrée une actualité plus large, qui englobe les mouvements de la politique.

En ce qui concerne plus précisément les premières lignes du reportage, une fois les textes introductifs passés, la typographie a été conservée: les nombreux retours à la ligne, qui coupent la lecture, imitent le regard étendu sur les paysages, restituant ainsi la primauté de la vue sur l'explication.

C’était Dakar!

Ce bloc de pierres blanches : le palais du gouverneur général. À notre droite: Gorée, l'île où les derniers négriers embarquaient les derniers esclaves sur un bâtiment qui s'appelait le Rendu.

Le Rendu qui ne rendait jamais rien ${ }^{46 !}$

Le style télégraphique de Londres - la deuxième phrase est nominale - reste celui du reporter qui recrée la rapidité de l'information, transmettant directement ce qu'il voit à son lecteur métropolitain. Mais quelques variations subsistent d'un support à un autre : ainsi, dans le livre, la mention de la première personne du pluriel aura l'effet d'inclure le lecteur, alors qu'elle ne signale, dans Le Petit Parisien, que la présence du photographe et dessinateur Georges Rouquayrol, toujours au second plan par rapport au journaliste. Ce changement qui affecte le pronom personnel modifie également la portée de la lecture, au sens où l'implication du lecteur semble plus grande dans le recueil : intégré à la narration, ayant la possibilité d'enchainer les épisodes de l'aventure, il est censé comprendre au sens fort les péripéties vécues par le reporter. Passé du journal au livre, le reporter maintient son image d'aventurier pressé par le temps; il parvient à conserver le rythme de l'actualité pour son lectorat de librairie, sorte de lectorat en différé qui ne s'installe pas dans la lecture de la même manière que lorsqu'il s'agit du journal. 
Ces remarques sur le rythme du reportage chez Albert Londres peuvent amener, par comparaison, à traiter rapidement d'un autre débarquement à Dakar, quelques années plus tard. Louis-Ferdinand Céline dans son Voyage au bout de la nuit décrit lui aussi cette scène topique qu'est l'arrivée à Dakar dans ces années 1930. Il se tient alors à proximité de Londres, comme Paul Aron le laisse entendre: "Rédigé à la première personne du singulier, par un narrateur qui est l'acteur de son récit, par un témoin qui nous fait croire à la véracité des faits rapportés [...], le récit de Céline se coule pleinement dans ce qu'on a décrit comme la matrice stylistique du grand reportage ${ }^{47}$. » Le voyage célinien se construit par épisodes successifs; mais chaque voyage se lit comme une partition isolée composant l'œuvre globale, et l'arrivée à Dakar vaut bien à ce titre comme un début d'épisode. Le voyage en Afrique de Bardamu s'amorce avec une rapidité marquée : le bateau sur lequel se focalise avant tout la description succède immédiatement au dialogue, sans autre transition.

Les huiles ont fini par me laisser tomber et j'ai pu sauver mes tripes, mais j'étais marqué à la tête et pour toujours. Rien à dire. «Va-t'en!... qu'ils m’ont fait. T’es plus bon à rien!...

- En Afrique! que j'ai dit moi. Plus que ça sera loin, mieux ça vaudra! »C'était un bateau de la Compagnie des Corsaires Réunis qui m'a embarqué. Il s'en allait vers les Tropiques, avec son fret de cotonnades, d'officiers et de fonctionnaires ${ }^{48}$.

Le bateau célinien est une métonymie de la société coloniale, usée et délétère; et là réside peut-être une clef de ces variations de rythme qui contraignent l'écriture du reportage : Londres n'a pas le loisir de décrire autant le bateau qui l'amène sur la terre africaine. En revanche, deux formules de cadence semblable et aux idées voisines réunissent ces textes : «Nous voguions vers l'Afrique, la vraie, la grande 49 » chez Céline fait écho au «C’était l'Afrique, la vraie, la maudite ${ }^{50} »$ de Londres, et dans les deux cas se lit la démythification de l'Afrique opérée au profit du lectorat métropolitain ${ }^{51}$. L'un se sert de la traversée pour formuler son attente de l'A frique stéréotypée, alors que l'autre formule cette attente à l'instant du débarquement, quand les côtes sont en vue; mais de part et d'autre se perçoivent également l'ironie, la distance manifestée entre le narrateur et son message par le biais d'un rythme ternaire. Plus largement, le lecteur retrouve dans les deux textes l'esquisse de passagers coloniaux ayant pour effet de mettre en lumière la subjectivité et la personnalité particulière du narrateur aventurier. Le rythme de lecture s'alentit 
dans les deux cas au moment d'évoquer le bateau et ses occupants; mais Céline décrit longuement la traversée alors que Londres ne réserve que quelques lignes à l'instantané du débarquement. La description de la traversée et des compagnons de voyage dans le roman célinien constitue l'axe par lequel Bardamu découvre vraiment la colonie, et l'anachronisme du bateau, ancien et décati, explique peut-être cette écriture qui s'apparente, avec originalité, à celle du journal de bord. Londres exploite lui aussi la présence des personnages coloniaux sur le bateau comme un avant-goût et un résumé du monde colonial à venir, mais l'exigence d'actualité du reportage lui fait accélérer le rythme, et l'idéologie coloniale explique alors que le bateau soit seulement une étape de la découverte. La différence de poétique visible entre les deux textes prend donc corps à partir du bateau qui inaugure le récit, et du rythme que les auteurs impriment à leur déplacement jusqu'en Afrique ${ }^{52}$.

\section{L’arrivée dans la colonie, un révélateur}

Les œuvres étudiées ici sont des récits de voyage, si l'on emploie cette expression comme un hyperonyme recouvrant les différentes réalisations des auteurs. Mais leurs supports de publication, ainsi que leurs contextes, ne se recoupent pas: récit de voyage présenté comme un document, roman d'aventures, reportage constituent les trois genres dans lesquels se réalisent les incursions dans les colonies, et ces genres correspondent à des formes littéraires prédominantes pour chacune des époques envisagées. La frontière souvent poreuse entre les genres du voyage montre cependant que le rapprochement de ces textes permet d'en souligner les différences, particulièrement pour les publications journalistiques où la présentation du texte vaut souvent comme carte d'identité générique. Dans cette même perspective, les premières lignes des textes évoqués, ou écritures liminaires, révèlent toutes quelque chose de la modernité imaginée au tournant du siècle et de ses variations : le thème colonial agit comme un catalyseur de problématiques à la fois idéologiques et esthétiques, chaque auteur inscrivant la scène de l'arrivée dans la colonie selon les modifications technologiques et littéraires de son époque.

S'il n'y a plus de colonies aujourd'hui, subsistent les voyages et leurs écritures: les seuils de ces voyages font-ils toujours la part belle au déplacement, à ces moments où se forgent l'instantanéité de la lecture et la 
portée de son rythme? Un court exemple servira ici de piste: Laurent Mauvignier inaugure un roman intitulé Autour du monde par une phrase comme "Quelle heure il peut être chez moi53? », sans que le lecteur soit transporté en même temps que le personnage. Il en résulte un sentiment d'abolition de la distance représentatif du lien actuel que l'écriture entretient avec le déplacement - ce que l'on peut appeler une poétique du déplacement, laquelle s'est modifiée au fur et à mesure que les technologies dudit déplacement ont évolué. La mise en scène de l'arrivée sur le lieu colonial et du moyen de transport apparait ainsi comme un impératif des récits, fictionnels ou non, écrits pendant la période d'expansion coloniale : représenter le trajet, c'est déjà donner une idée de la colonisation. D’où le fait que Céline arrive si bien à contourner la description des personnages (ce que nous apprend la confrontation de son écriture avec celle de Londres); d'où le fait que cette poétique du déplacement se soit perdue avec l'enthousiasme colonial. Le support médiatique, même quand il était complété par une publication en livre, travaillait en sourdine ces représentations de l'aventure et leurs débuts : là encore, la modification de l'environnement des publications a ouvert la voie à d'autres poétiques, qui ont à voir avec l'invention d'internet et sa remise en question des rythmes culturels.

Agrégée de lettres modernes, Laure Demougin a récemment soutenu une thèse intitulée «Identités et exotisme : représentation de soi et des autres dans la presse coloniale française au xix ${ }^{e}$ siècle », sous la direction de Marie-Ève Thérenty (Université Paul-Valéry Montpellier 3) et Guillaume Pinson (Université Laval). Ses travaux portent principalement sur la presse coloniale et sur la littérature postcoloniale.

\section{Notes}

${ }^{1}$ En ce qui concerne « l'aventure coloniale », voir le $n^{\circ} 7$ des Cabiers de la SIELEC, dirigé par Jean-François Durand et Jean-Marie Seillan, et plus particulièrement l'introduction rédigée par Sylvain Venayre.

2 Romain Bertrand, "Les sciences sociales et le "moment colonial" : de la problématique de la domination coloniale à celle de l'hégémonie impériale », Questions de recherche / Research in Question, n 18, 2006. https://hal-sciencespo.archives-ouvertes.fr/hal-01065637/document. 
${ }^{3}$ Nous nous appuyons ici sur l'ouvrage de François Hartog, Régimes d'historicité. Présentisme et expérience du temps, Paris, Seuil, 2008 [2003]. Il y définit notamment le régime d'historicité comme la «modalité de conscience de soi d’une communauté humaine » (p. 29) : le régime d'historicité colonial fait prédominer l'avenir sur le présent ou le passé, orientant ainsi toute la perspective temporelle vers une forme de linéarité.

${ }^{4}$ Voir Mélodie Simard-Houde, Le reporter et ses fictions. Poétique historique d'un imaginaire, Limoges, PULIM, coll. « Médiatextes », 2017.

${ }^{5}$ Voir Marie-Ève Thérenty, La littérature au quotidien. Poétiques journalistiques an XIX siècle, Paris, Seuil, 2007.

${ }^{6}$ Marie-Ève Thérenty, La littérature au quotidien. Poétiques journalistiques an XIXe siècle, Paris, Seuil, 2007, p. 295.

${ }^{7}$ Sylvain Venayre, «Le voyage, le journal et les joumalistes au XIX ${ }^{\mathrm{e}}$ siècle», Le Temps des médias, $\mathrm{n}^{\circ} 8$, 2007, pp. 46-56.

${ }^{8}$ Myriam Boucharenc, «Choses vues, choses lues : le reportage à l'épreuve de l'intertexte", Cahiers de Narratologie, n 13, 2006, p. 4.

${ }_{9}^{9}$ Arthur Delteil, « Voyage chez les Indiens de la Guyane », La Fexille de la Guyane francaise, 27 mai 1871.

10 Arthur Delteil, «Voyage chez les Indiens de la Guyane », La Fexille de la Guyane française, 27 mai 1871.

11 Arthur Delteil, «Voyage chez les Indiens de la Guyane », La Fexille de la Guyane française, 27 mai 1871.

12 Selon la définition du Trésor de la Langue Française. http://www.cnrtl.fr/definition/goelette.

13 Arthur Delteil, «Voyage chez les Indiens de la Guyane», La Ferille de la Guyane française, 27 mai 1871.

14 Arthur Delteil, «Voyage chez les Indiens de la Guyane », La Fenille de la Guyane française, 27 mai 1871.

15 Arthur Delteil, «Voyage chez les Indiens de la Guyane », La Feuille de la Guyane française, 27 mai 1871.

16 Références : Arthur Delteil, Étude sur la vanille, Paris, Challamel aîné, 1874; La Canne à sucre, Paris, Challamel aîné, 1884; Étude sur le climat de la Cocbinchine, Nantes, L. Mellinet, 1885; Note sur le climat de la Réunion, Paris, Challamel aîné, 1886.

${ }_{17}$ Arthur Delteil, Voyage chę les indiens Galibis de la Guyane, Nantes, L. Mellinet, 1886, p. 1.

18 Voir les problématiques du numéro de Mémoires du liure dirigé par Adrien Rannaud, «Le livre et le journal : croisements, prolongements et transformations», vol. 8, $\mathrm{n}^{\circ}$ 2, printemps 2017. https://www.eruditorg/fr/revues/memoires/2017-v8-n2-memoires03051/.

Vol. 10, $\mathrm{n}^{\circ} 1 \mid$ Automne 2018

«Voyager dans la modernité : des moyens techniques aux supports imprimés de l'aventure » 
${ }^{19}$ Lionel Dupuy, «Les Voyages extraordinaires de Jules Verne ou le roman géographique au XIXe siècle », Annales de géographie, nº 690, 2013, pp. 131-150.

${ }^{20}$ Sylvain Venayre, «Le voyage, le journal et les journalistes au XIX siècle», Le Temps des médias, $n^{\circ} 8$, 2007, pp. 46-56.

${ }^{21}$ Guillaume Pinson, "Irkoutsk ne répond plus. Jules Verne, les médias de masse et l'imaginaire de la rupture de communication », Romantisme, vol. 4, n 158, 2012, pp. 83-96.

22 Voir Edward Ousselin, "De Nemo à Dakkar. La représentation paradoxale du colonialisme chez Jules Verne », Nineteenth-Century French Studies, vol. 42, n 1-2, 2013-2014, pp. 88-102.

${ }^{23}$ Le Temps, 20 décembre 1896, supplément.

${ }^{24}$ Jules Verne, Face au drapean, Paris, Hetzel, 1896, p. 1.

${ }^{25}$ Jules Verne, Clovis Dardentor, Paris, Hetzel, 1896.

${ }^{26}$ Jules Verne, Clovis Dardentor, Paris, Hetzel, 1896, p. 1.

${ }^{27}$ Alphonse Daudet, Les Aventures prodigieuses de Tartarin de Tarascon, Paris, Dentu, 1872. Voir aussi la «causerie littéraire » parue en feuilleton le 26 décembre 1896 dans Le Gaulois et le 28 décembre 1896 dans Le Journal : l'auteur (qui signe L. O.) trace le parallèle entre les deux méridionaux que sont Tartarin et Dardentor.

28 Jules Verne, Clovis Dardentor, Paris, Hetzel, 1896, p. 1.

${ }^{29}$ Myriam Boucharenc, L'écrivain-reporter au cour des années trente, Villeneuve d'Ascq, Presses universitaires du Septentrion, 2004, p. 27.

${ }^{30}$ Élie-J. Bois, Le Petit Parisien, 11 octobre 1928.

${ }^{31}$ Élie-J. Bois, Le Petit Parisien, 11 octobre 1928.

32 Albert Londres, «Quatre mois parmi nos noirs d'Afrique », Le Petit Parisien, 12 octobre 1928.

${ }_{33}$ Mélodie Simard-Houde, Le reporter et ses fictions. Poétique historique d'un imaginaire, Limoges, PULIM, coll. « Médiatextes », 2017.

${ }^{34}$ Mélodie Simard-Houde, «Le reporter, médiateur, écrivain et héros. Un répertoire culturel (1870-1939) ", thèse de doctorat, Québec, Université Laval, Département de littérature, théâtre et cinéma, 2015.

35 Sylvain Venayre, «Le voyage, le journal et les journalistes au XIXe siècle», Le Temps des médias, $\mathrm{n}^{\circ} 8$, 2007 , p. 54.

${ }^{36}$ Des variations dans la mise en page permettent aux éditeurs de jouer sur la rapidité de la lecture : dans l'édition Arléa de 1992, un retour à la ligne entre "Six heures! on accroche la

Vol. 10, $\mathrm{n}^{\circ} 1 \mid$ Automne 2018

«Voyager dans la modernité : des moyens techniques aux supports imprimés de l'aventure » 
passerelle au bateau» et la phrase suivante : «Les fonctionnaires coloniaux sentent une angoisse les pincer au cœur » (p. 16) accélère encore le rythme de lecture.

${ }^{37}$ Albert Londres, Terre d'ébène (la traite des noirs), Paris, Albin Michel, 1929, p. 58.

38 Albert Londres, «Quatre mois parmi nos noirs d'Afrique », Le Petit Parisien, 18 octobre 1928.

39 Albert Londres, Terre d'ébène (la traite des noirs), Paris, Albin Michel, 1929, p. 58.

40 Albert Londres, Terre d'ébène (la traite des noirs), Paris, Albin Michel, 1929, p. 140. Paru le 31 octobre 1928.

${ }^{41}$ Albert Londres, Terre d'ébène (la traite des noirs), Paris, Albin Michel, 1929, p. 128. Paru le 26 octobre 1928.

42 Albert Londres, Terre d'ébène (la traite des noirs), Paris, Albin Michel, 1929, p. 91.

43 Albert Londres, «Quatre mois parmi nos noirs d'Afrique », Le Petit Parisien, 22 octobre 1928.

${ }^{44}$ Frédéric Lambert, dans " Esthésie de la dénonciation. Albert Londres en Terre d'ébène ", Le Temps des médias, $\mathrm{n}^{\circ}$ 26, 2016, parle ainsi des « vivacités » du texte de Londres (p. 75).

45 Albert Londres, Terre d'ébène (la traite des noirs), Paris, Albin Michel, 1929, p. 5. C'est dans cette préface que Londres exprime ce qui deviendra un véritable leitmotiv journalistique : «Notre métier n'est pas de faire plaisir, non plus de faire du tort, il est de porter la plume dans la plaie » (p. 6).

46 Albert Londres, «Quatre mois parmi nos noirs d'Afrique », Le Petit Parisien, 12 octobre 1928.

47 Paul Aron, «Entre journalisme et littérature, l'institution du reportage», COnTEXTES, n 11, 2012. http://contextes.revues.org/5355.

${ }^{48}$ Louis-Ferdinand Céline, Voyage au bout de la nuit, Paris, Gallimard, coll. « Pléiade », 1952, p. 111.

${ }^{49}$ Louis-Ferdinand Céline, Voyage au bout de la nuit, Paris, Gallimard, coll. «Pléiade », 1952, p. 112.

50 Albert Londres, «Quatre mois parmi nos noirs d'Afrique », Le Petit Parisien, 12 octobre 1928.

${ }^{51}$ En ce qui concerne «Quatre mois parmi nos noirs d'Afrique », voir l'article de Sophie Desmoulin, "Quatre mois parmi nos Noirs d'Afrique" à la une du Petit Parisien: représentations de l'Afrique et de l'Africain à la fin des années 1920 », Le Temps des médias, n²6, 2016, p. 64.

${ }^{52}$ D’où le fait, malgré la parenté stylistique du roman célinien et du reportage, qu'on puisse qualifier Céline d'antireporter, comme le fait Mélodie Simard-Houde dans l'un de ses 
articles: «Les avatars du "Je". Roman et reportage dans l'entre-deux-guerres », Études françaises, vol. 52, $\mathrm{n}^{\circ} 2,2016$, pp. 161-180.

${ }^{53}$ Laurent Mauvignier, Autour du monde, Paris, Minuit, 2014.

\section{Bibliographie}

\section{Sources}

Louis-Ferdinand Céline, Voyage au bout de la nuit, Paris, Gallimard, coll. «Pléiade », 1952.

Alphonse Daudet, Les Aventures prodigieuses de Tartarin de Tarascon, Paris, Dentu, 1872.

Arthur Delteil, Voyage chez. les indiens Galibis de la Guyane, Nantes, Mellinet, 1886.

Arthur Delteil, "Voyage chez les Indiens de la Guyane », La Feuille de la Guyane française, du 27 mai au 17 juin 1871.

Albert Londres, Terre d'ébène (La traite des noirs), Paris, Albin Michel, 1929.

Albert Londres, "Quatre mois parmi nos noirs d'Afrique », Le Petit Parisien, du 12 octobre au 11 novembre 1928.

Laurent Mauvignier, Autour du monde, Paris, Minuit, 2014.

Jules Verne, Clovis Dardentor, Paris, Hetzel, 1896.

Jules Verne, Face an drapeau, Paris, Hetzel, 1896.

\section{Ouvrages et articles}

Paul Aron, «Entre journalisme et littérature, l'institution du reportage », COnTEXTES, n 11, 2012. http://contextes.revues.org/5355.

Romain Bertrand, "Les sciences sociales et le "moment colonial" : de la problématique de la domination coloniale à celle de l'hégémonie impériale », Questions de recherche / Research in Question, $\mathrm{n}^{\circ}$ 18, 2006. https://halsciencespo.archives-ouvertes.fr/hal-01065637/document.

Myriam Boucharenc, L'écrivain-reporter au coeur des années trente, Villeneuve d'Ascq, Presses universitaires du Septentrion, 2004.

Myriam Boucharenc, "Choses vues, choses lues : le reportage à l'épreuve de l'intertexte », Cabiers de Narratologie, n 13, 2006. http:// narratologie.revues.org/320 (26 septembre 2018). 
Sophie Desmoulin, " "Quatre mois parmi nos Noirs d'Afrique" à la une du Petit Parisien: représentations de l'Afrique et de l'Africain à la fin des années 1920 », Le Temps des médias, $\mathrm{n}^{\circ} 26,2016$, pp. 57-74.

Lionel Dupuy, «Les Voyages extraordinaires de Jules Verne ou le roman géographique au XIX siècle », Annales de géographie, nº 690, 2013, pp. 131-150.

Jean-François Durand et Jean-Marie Seillan (dir.), Cahiers de la SIELEC. L'aventure coloniale, Paris, Kailash, 2011.

Gérard Genette, Figures III, Paris, Seuil, 1972.

François Hartog, Régimes d'bistoricité. Présentisme et expérience du temps, Paris, Seuil, 2008 [2003].

Frédéric Lambert, «Esthésie de la dénonciation. Albert Londres en Terre d'ébène », Le Temps des médias, $\mathrm{n}^{\circ}$ 26, 2016, pp. 75-92.

Edward Ousselin, «De Nemo à Dakkar. La représentation paradoxale du colonialisme chez Jules Verne », Nineteenth-Century French Studies, vol. 42, n ${ }^{\circ} 1-2$, 2013-2014, pp. 88-102.

Guillaume Pinson, «Irkoutsk ne répond plus. Jules Verne, les médias de masse et l'imaginaire de la rupture de communication », Romantisme, vol. 4, $\mathrm{n}^{\circ}$ 158, 2012, pp. 83-96.

Mélodie Simard-Houde, "Les avatars du "Je". Roman et reportage dans l'entredeux-guerres », Études françaises, vol. 52, n² 2, 2016, pp. 161-180.

Mélodie Simard-Houde, Le reporter et ses fictions. Poétique historique d'un imaginaire, Limoges, PULIM, coll. « Médiatextes », 2017.

Mélodie Simard-Houde, «Le reporter, médiateur, écrivain et héros. Un répertoire culturel (1870-1939) », thèse de doctorat, Québec, Université Laval, Département de littérature, théâtre et cinéma, 2015.

Marie-Ève Thérenty, La littérature au quotidien. Poétiques journalistiques au XIX siècle, Paris, Seuil, 2007.

Sylvain Venayre, «Le voyage, le journal et les journalistes au XIX ${ }^{\mathrm{e}}$ siècle », Le Temps des médias, $\mathrm{n}^{\circ}$ 8, 2007, pp. 46-56. 